\title{
Correspondence
}

\author{
Letters should not exceed 400 words and should be typed double spaced (including the references) and be signed by all authors
}

TO THE EDITOR, Genitourinary Medicine

\section{Genital warts and the need for screening}

Sir,

It is believed among genitourinary physicians that the presence of genital warts in women warrants screening for other sexually transmitted diseases (STDs). This view was supported by a study by Kinghorn, ${ }^{1}$ in which he concluded that there was a relatively high incidence of other genital infections in women suffering from genital warts. At the time of this study routine tests for chlamydia were not available. The changing patterns of STD incidence over recent years might be expected to alter the risk of associated infections.

We recently reviewed 100 consecutive women attending our department with genital warts, all of whom were screened for other STDs. All were screened using standard methods for Neisseria gonorrhoea, Candida albicans, Trichomonas vaginalis and syphilis. Cervical swabs were tested for identification of Chlamydia trachomatis by micro-immunofluorescence. "Bacterial vaginosis" was diagnosed in women with a symptomatic malodourous vaginal discharge, and in whom microscopy of vaginal samples revealed "clue cells" or abnormal flora.

No women had positive syphilis serology or culture for gonorrhoea. The numbers (percentages) of women with positive diagnoses is shown in the table.

We found a lower than expected incidence of associated STDs in women with genital warts. Although a number of authors have concluded that there is an increased incidence of other genital tract infections in

Table Incidence of associated lower genital tract infection in women presenting with genital warts $(n=100)$

Chlamydia trachomatis

Symptomatic candida

Bacterial vaginosis

Trichomonas*

Any of the above

Asymptomatic candida only

No associated infection found

*The single woman with trichomonas was also positive for chlamydia. women presenting with genital warts, they have largely failed to show a high incidence of STDs.

Kinghorn' highlighted in his study the significant proportion of women who had other genital infections. However the main infective agent discovered in his study was Candida albicans; there were in fact only $12.3 \%$ of women with gonorrhoea and $0.9 \%$ with "non-specific genital infection". Statistics from returns to the DHSS show that the incidence of gonorrhoea has fallen significantly since a peak in $1973 .^{2}$ It may well be that the apparent absence of an association between the presence of genital warts and this infection is a reflection of the decreased incidence in the general population. In our study only a single case of trichomonas vaginitis was found.

Longhurst and colleagues ${ }^{3}$ studied a group of women in a north London general practice finding a $10.7 \%$ rate of chlamydia infection, by micro-immunofluorescence. Turner et al ${ }^{4}$ reporting on STD screening in a group of women attending for colposcopy after an abnormal smear showed $9 \%$ to have infection with chlamydia, $2 \%$ had trichomonas and $3 \%$ had positive syphilis serology.

It appears that the incidence of other STDs is no higher in an unselected group of women with genital warts than it would be among young sexually active women in general. A blanket policy of screening all women with genital warts for other sexually transmitted diseases, whilst ignoring a similar risk in women without warts may seem inappropriate. Further research might seek to establish particular high risk groups for selective screening.

\section{Malcolm Griffiths And on behalf of Lynn J Overington and Sue $C$ Chard}

Dept of Genitourinary Medicine,

Lloyd Clinic, Guy's Hospital,

London SE1 9RT, UK

\section{References}

1 Kinghorn GR. "Genital warts: incidence of associated genital infections." Br J Dermatol 1978;99:405-9.
2 Communicable Disease Surveillance Centre. "Sexually transmitted disease surveillance in Britain-1984." Br Med J 1986;293:942-3.

3 Longhurst LJ, Flower N, Thomas BJ et al. A simple method for detection of Chlamydia trachomatis infection in general practice. $J \boldsymbol{R}$ Coll Gen Pract 1987;37:1255-6.

4 Turner MJ, White JO, Soutter WP. "The male factor in cervical neoplasia." Contemp Rev Obstet Gynaecol 1988;1:36-42.

TO THE EDITOR, Genitourinary Medicine

\section{Trichomonas vaginalis infection in a lesbian}

Sir,

We report a case of Trichomonas vaginalis infection in a lesbian. The 25 year old single Caucasian female was referred to our department with a 4 month history of offensive vaginal discharge and pruritus vulvae. She developed these symptoms after having sexual relationship with a casual bisexual girlfriend in London in December 1988. She denied sharing vibrators or sex toys, but admitted using her fingers for masturbating her partner and herself. She had gonorrhoea following heterosexual intercourse in September 1982 which helped her to change to homosexual practice, rather than initiate it. She had no STDs other than her gonorrhoea infection which responded successfully to treatment. The patient's sailor consort at that time, was treated too. She vehemently denied further heterosexual intercourse or contact since 1982.

On examination she had moderate erythema of the vulva with yellowish green frothy vaginal discharge suggestive of Trichomonas infection. Immediate wet drop examination of the discharge collected from the posterior fornix of her vagina confirmed numerous Trichomonas vaginalis protozoon (TV) and the clinical/microscopic diagnosis was confirmed by culture using oxoid trichomonas media. Smears and cultures for candida, gonorrhoea, Chlamydia trachomatis and the serological tests for syphilis were negative. The patient was treated with a single $2.0 \mathrm{~g}$ oral dose of metronidazole following which she had an excellent response and had two 
sets of negative tests for TV and other STDs at one and three week intervals following treatment. Her casual consort in London was untraceable.

$T$. vaginalis is a cosmopolitan flagellate of the genital organs in adults. Transmission occurs primarily through sexual intercourse as the parasite has no resistant stage. Infection is commonly symptomless in the male and produces a severe vaginitis in the female. There is little evidence to support direct female to female transmission resulting from poor standards of sanitation and hygiene. Whittington' showed that the Trichomonas vaginalis organism in vaginal exudate can survive up to 48 hours when maintained at $10^{\circ} \mathrm{C}$. Hesseltine et al $(1942)^{2}$ produced the clinical entity of vaginal trichomoniasis by innoculation of the human vagina with vaginal trichomonads.

We believe this is the first reported case of Trichomonas vaginalis infection acquired probably in a lesbian relationship transmitted from partner's vaginal exudates through masturbating fingers.

Dept of Genitourinary Medicine,

K Sivakumar

A H De Silva

$R$ Basu Roy

Royal Victoria Hospital,

Gloucester Road,

Bournemouth BH7 6JF, UK

\section{References}

1 Whittington MJ. The survival of Trichomonas vaginalis at temperatures below $37^{\circ} \mathrm{C} . \mathrm{J} \mathrm{Hyg}$ Epidemiol Microbiol Immunol 1951;49:400.

2 Hesseltine HC, Wolters SL, Campbell A. Experimental human vaginal trichomoniasis. J Infect Dis 1942;127:71.

\section{TO THE EDITOR, Genitourinary Medicine}

Falling prevalence of Chlamydia trachomatis infection among female patients attending the Department of Genito-Urinary Medicine, Bournemouth

Sir,

Over the last 5 years we have noticed a significant fall in the prevalence of chlamydia infection among female patients attending our department. It has fallen from $14.57 \%$ culture positives in 1984 to $3.23 \%$ in the first six months of 1989. A national fall in the prevalence of syphilis, gonorrhoea and trichomonas vaginalis infections have been reported before. ${ }^{1}$ We feel this fall is mainly due to change of sexual behaviour as a result of the AIDS epidemic, health education, effective treatment, contact tracing and epidemiological treatment of female consorts of male patients with non-specific urethritis. Neisseria gonorrhoeae and particularly Chlamydia trachomatis cause $40-70 \%$ of pelvic inflammatory disease in the western world. ${ }^{2-3}$ If this trend in the falling prevalence of Chlamydia trachomatis and Neisseria gonorrhoeae infections continues, we expect to see fewer cases of pelvic inflammatory disease as well as its complications in the future. The falling incidence of sexually transmitted diseases, particularly of Trachomonas vaginalis, gonorrhoea, Chlamydia trachomatis, confirms that the heterosexuals also, have modified their sexual behavioural response, contrary to reports in the national press.

\section{K Sivakumar} $R$ Basu Roy

Dept of Genitourinary Medicine,

Royal Victoria Hospital,

Gloucester Road,

Bournemouth BH7 6JF, UK

References

1 The Director, PHLS Communicable Disease Surveillance Centre-sexually transmitted disease in Britain 1985/6. Genitourin Med 1989;65:117-21.

2 Cunningham FG, Hauth JC, Gilstrap LC et al. The bacterial pathogenesis of acute pelvic inflammatory disease. Obstet Gynecol 1978;52:161.

3 Westrom L. Incidence, prevalence and trends of acute pelvic inflammatory disease and its consequences in industrialised countries. Am J Obstet Gynecol 1980;138:880.

TO THE EDITOR, Genitourinary Medicine

Declining incidence of Chlamydia trachomatis in women attending a provincial genitourinary medicine clinic

Sir,

Endocervical cultures for Chlamydia

Table

\begin{tabular}{lrrrr}
\hline Year & 1985 & 1986 & 1987 & 1988 \\
\hline Total new attenders & 3121 & 3205 & 3570 & 3156 \\
Number screened & 3063 & 3082 & 3326 & 3019 \\
Gonococcal isolates & 296 & 256 & 158 & 105 \\
C trachomatis isolates & 534 & 439 & 426 & 215 \\
\hline
\end{tabular}

trachomatis and Neisseria gonorrhoeas are offered to all women attending the Department of Genitourinary Medicine $\overrightarrow{\mathbf{F}^{\prime}}$ Newcastle. In a retrospective study the incidence of these two infections in womer during 1985 to 1988 were compared shown in the table. The new isolates of trachomatis and $N$ gonorrhoeae declines steadily over the four year period, while the number of women screened for these tw8 infections remained fairly stable. The incidence of Chlamydia trachomatis has fallen from $174 / 1000$ in 1985 to $71 / 1000$ igy 1988. A similar decrease in gonorrhoea has already been reported in London.' Ouf. observations in Newcastle show a paralle trend in these two sexually transmitted infections which are acquired by unprotectef penetrative sexual intercourse. These findo ings are suggestive of changes in sexuß behaviour in women attending a genito urinary medicine clinic.

K Shanmugaratnars R S PattmaA

Dept of Genitourinary Medicine,

Newcastle General Hospital,

Newcastle upon Tyne, NE4 6BE, UK

Reference

1 Gellan MCA, Ison CA. Declining incidence gonorrhoea in London: a response to fear AIDS. Lancet 1986;ii:920. 